\title{
The Comparison of Deficits in the Theory of Mind in Patients with Bipolar Disorder Type 1 in Remission Period
}

\section{Ebrahim Soltani Azemat ${ }^{1}$, Abolfazl Mohammadian' ${ }^{1}$, Behrooz Doolatshahee ${ }^{1^{*}}$, Hiro Alidost ${ }^{2}$}

${ }^{1}$ Department of Clinical Psychology, University of Social Welfare and Rehabilitation Sciences, Tehran, Iran

${ }^{2}$ Department of Clinical Psychology, Science and Research Branch, Islamic Azad University of Sanandaj, Sanandaj, Iran

\section{A BSTRACT}

Introduction: Recent studies on theory of mind (ToM) in patients with bipolar disorder have revealed deficits of ToM ability during episodes. This study was aimed to assess deficits in levels of ToM in patients with bipolar disorder type 1 in remission Period. Materials and Methods: Thirty men with bipolar disorder and 30 healthy men were selected by available sampling method. The questionnaires included Kessler Psychological Distress Scale (K10) and theory of mind picture stories task. Results: Our results indicated a significant difference between bipolar disorder patients vs. healthy individuals with regard to the total score of theory of mind picture stories task. Regarding ToM's levels, there was a significant difference between bipolar disorder patients and healthy individuals in five subscales included: second-order false beliefs, third-order false beliefs, reciprocity, deception, and detection of cheating. However, data indicated that there is no significant difference in first-order false belief. Conclusion: Bipolar patients spent their remission period showed a weaker performance in complex ToM's levels and false belief than healthy people. They were weak in cognitive understanding of others' mind states, like opinions and ambitions, and emotional mind states, like feelings and emotions. The deficit in complex levels of ToM in bipolar patients in remission period can be the result of the malfunction of cognitive infrastructures in the brain.

\section{Key words:}

1. Bipolar Disorder

2. Theory of Mind

3. Patients

*Corresponding Author: Behrooz Doolatshahee

E-mail:dolatshahee@yahoo.com 


\title{
مقايسةُ نقصها در نظريةٌ ذهن در بيماران با اختلال دو قطبى نوع ا در دوره فروكش
}

\author{
ابراهيم سلطانى عظمت'، ابوالفضل محمديان' ، بهروز دولتشاهى '، هيرو عليدوست' \\ 'كروه روانشناسى بالينى، دانشعاه علوم بهزيستى و توانبخشى، تهران، ايران \\ "كروه روانشناسى بالينى، واحد علوم و تحقيقات، دانشعاه آزاد اسلامى سنندج، سنندج، ايران
}

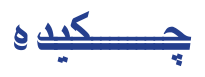

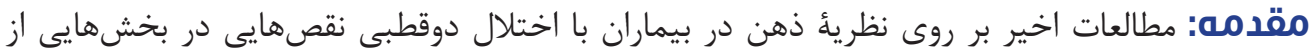

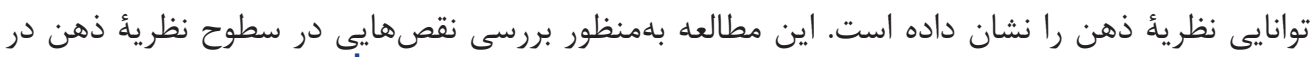

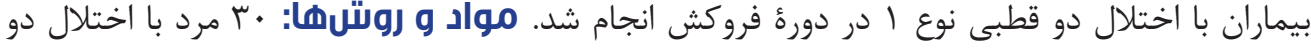

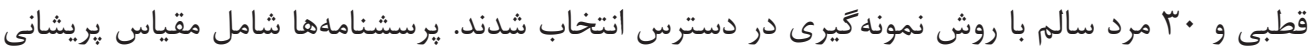

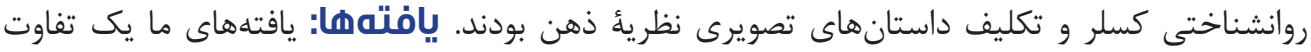

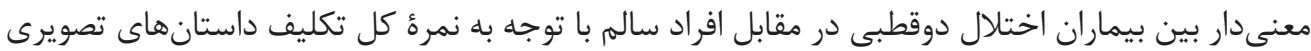

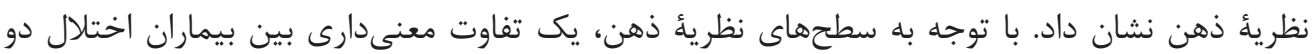

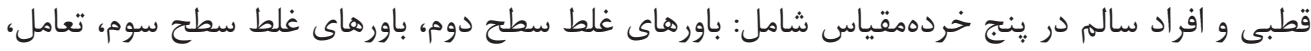

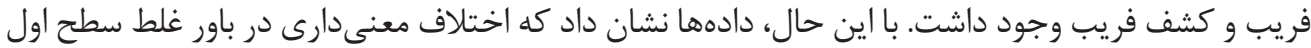

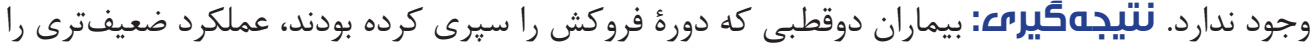

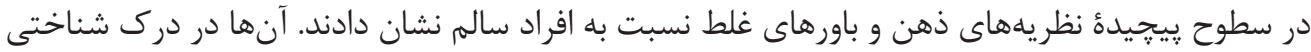

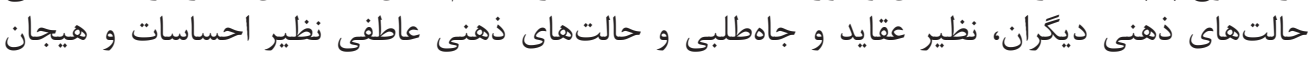

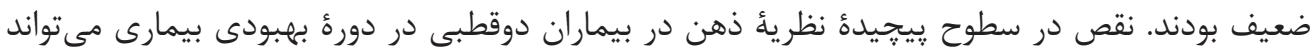

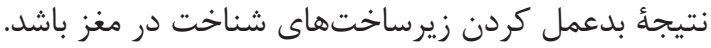

كليد وازهها:

ا I. اختلال دوقطبى

r. نظرئ ذهن

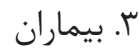

" نويسنده مسئول: بهروز دولتشاهى

آدرس الكترونيكى: dolatshahee@yahoo.com 
سطح اول در حدود ه-أ سالكَى ايجاد مىشود و باور غلط سطح

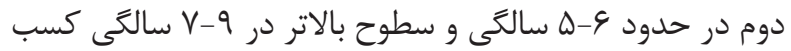

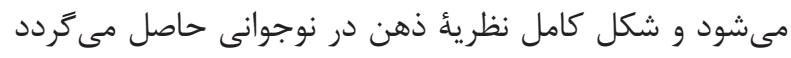

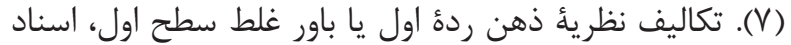

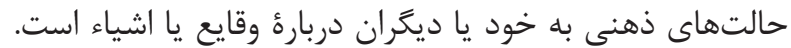

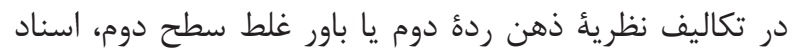

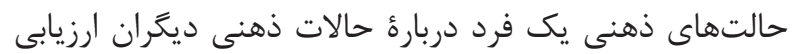

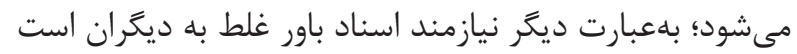

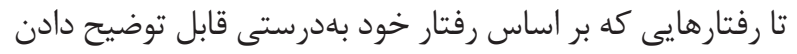

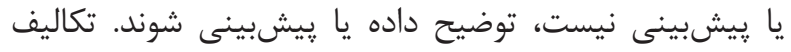

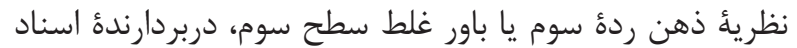

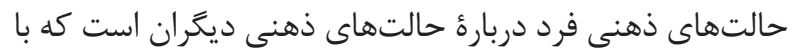
ساير حالتهاى ذهنى ارتباط دارد (^).

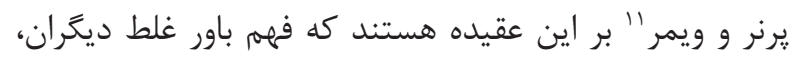

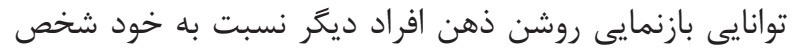

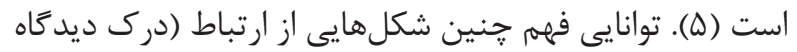

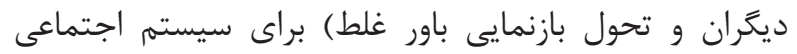

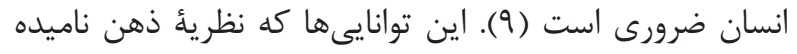

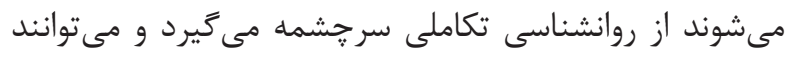
جنبههايى از آسيبشناسى روانى را روشن كنند (9). در طى دهأ كَذشته، مشاهده شده است كه نقايص نظرئ

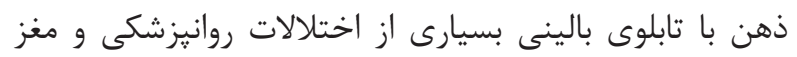

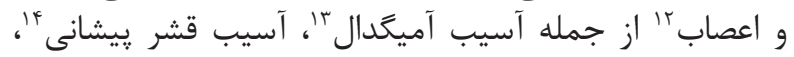

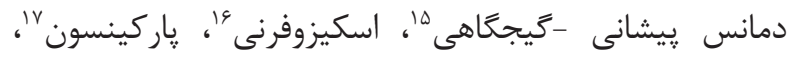

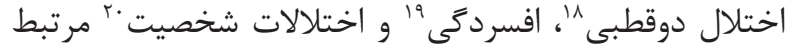

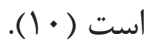

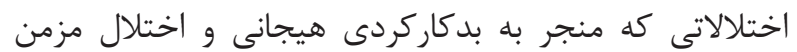

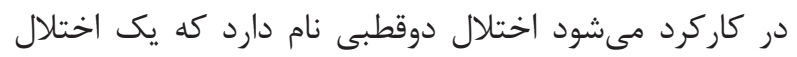

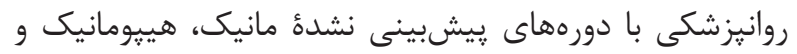

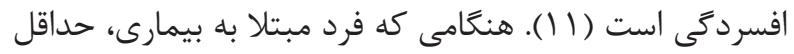

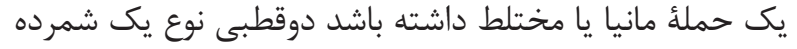

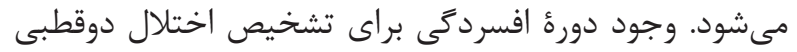

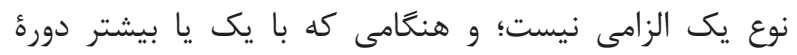

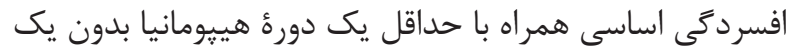

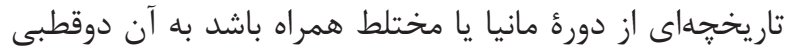
نوع دوم اطلاق مى كردد (1) (I).

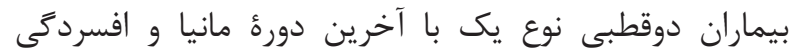

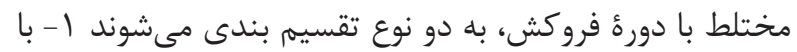

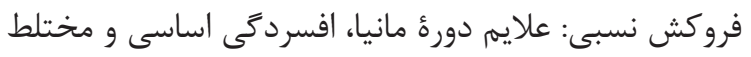

${ }^{1}$ Theory of mind

${ }^{2}$ Premack and Woodruff

${ }^{3}$ Mental states

${ }^{4}$ Desires

${ }^{5}$ Beliefs

${ }^{6}$ Intentions

${ }^{7}$ False belief

${ }^{8}$ First-order false belief

${ }^{9}$ Second-order false belief

${ }^{10}$ Third-order false belief

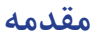

درك خود و ديكران براى داشتن تعاملات اجتماعى مؤثر، ضرورى دورى

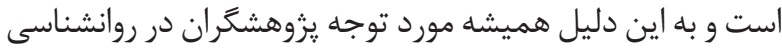

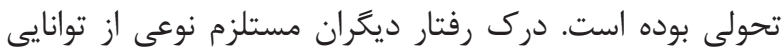

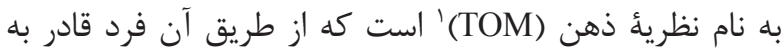

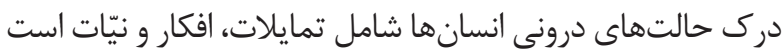

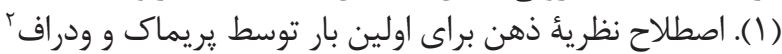

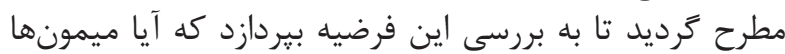

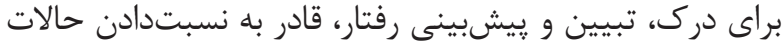

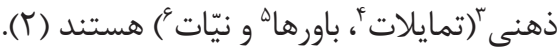

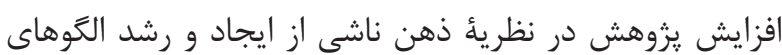

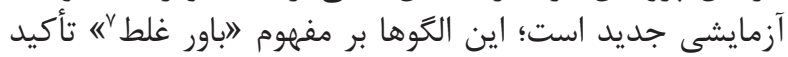

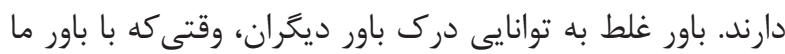

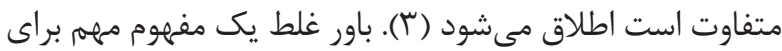

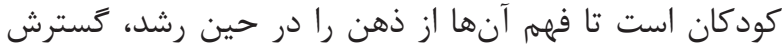

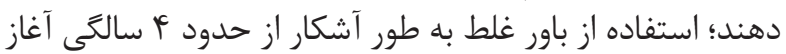

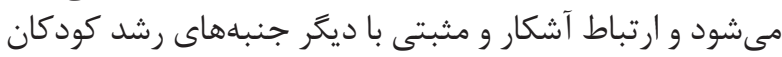

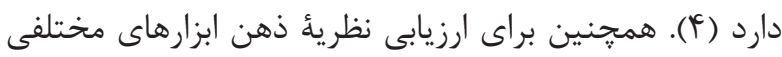

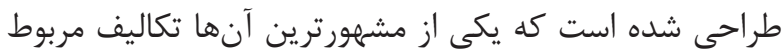

به باور غلط است (ه). (ه).

بهاعنوان نمونه، در يك تكليف معمول از باور غلط كه به تكليف

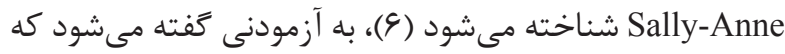
Sally

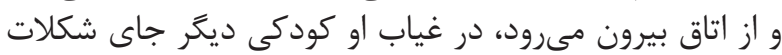

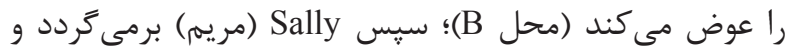

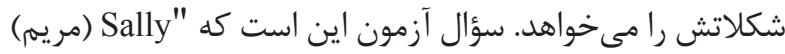

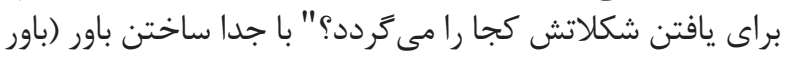

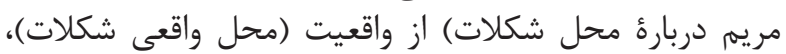

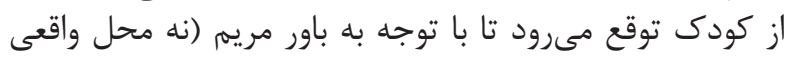

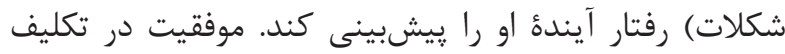

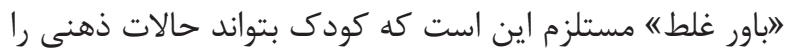

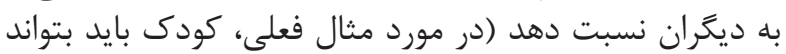

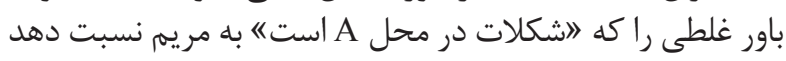

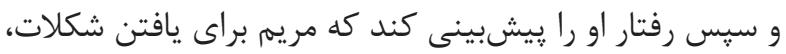

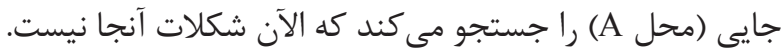

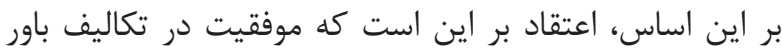

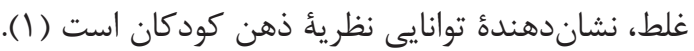

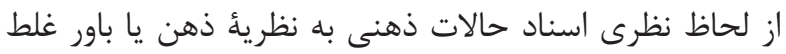

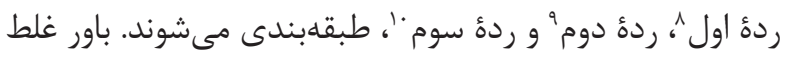

\footnotetext{
${ }^{11}$ Perner and Wimmer

${ }^{12}$ Neurological

${ }^{13}$ Amygdala

${ }^{14}$ Frontal damage

${ }^{15}$ Frontotemporal

${ }^{16}$ Schizophrenia

${ }^{17}$ Parkinson's disease

${ }^{18}$ Bipolar disorder

${ }^{19}$ Depression

${ }^{20}$ Personality disorders
} 


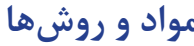

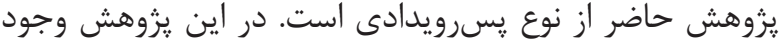

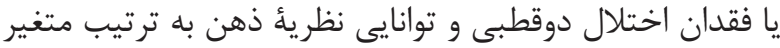

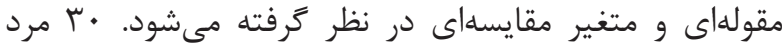

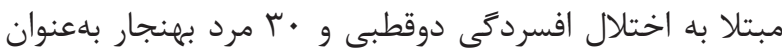

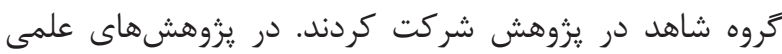

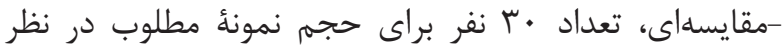

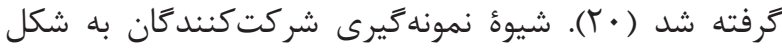

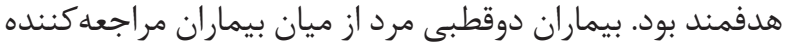

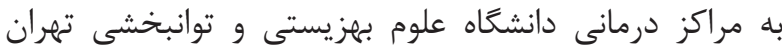

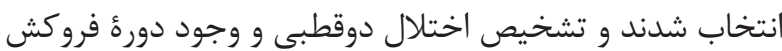

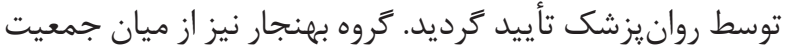

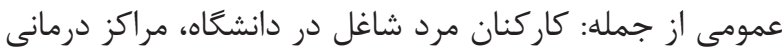

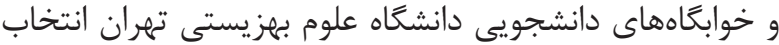

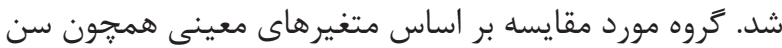

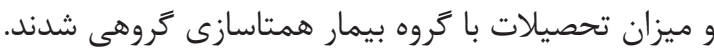

معيارهاى ورود براى شركت كنندكان هر دو كروه بيمار و و هنجار

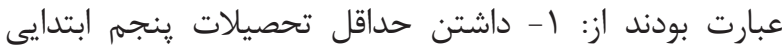

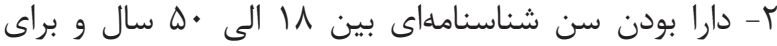
شركت كنند

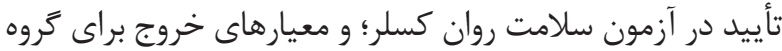

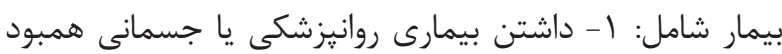

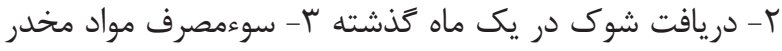

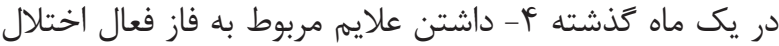

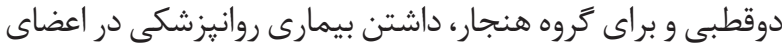

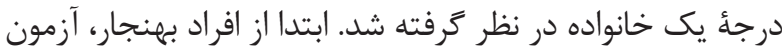

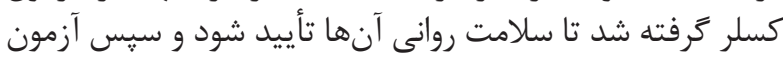

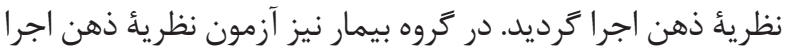

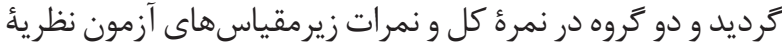

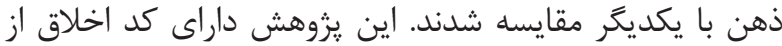

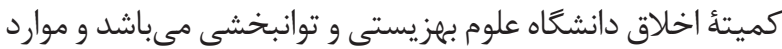

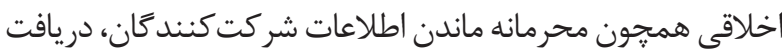

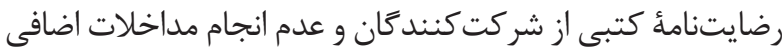

رعايت شده است.

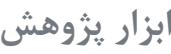

\section{تكليف داستانهاى تصويرى نظريئ ذهن}

كركرانه و همكاران براى ارزيابى داستانهاى تصويرى نظرئ

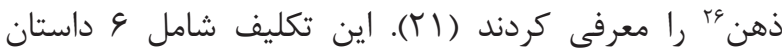

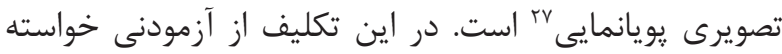

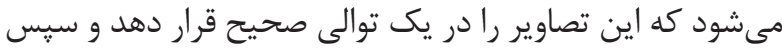

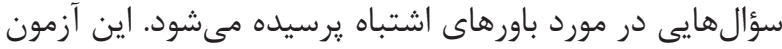

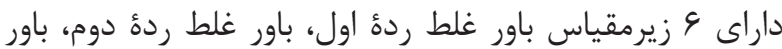

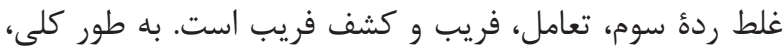

\footnotetext{
${ }^{21}$ Prefrontal

${ }^{22}$ Cingulate gyrus

${ }^{23}$ Samamé

${ }^{24}$ Tonelli
}

وجود داشته باشد اما نه همأ ملاكها و يا يا در يك دور دورة زمانى

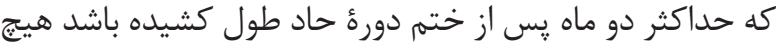

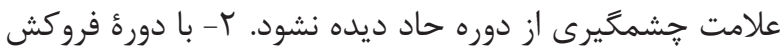

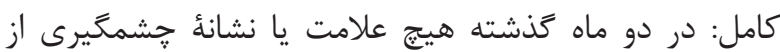

اختلال وجود نداشته باشد (T) (1).

از مشخصههاى بارز بيماران دوقطبى، اختلال در عملكرد

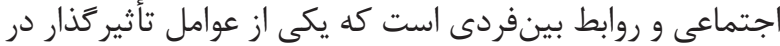

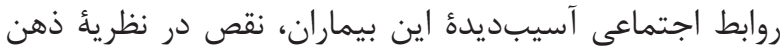

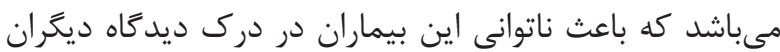

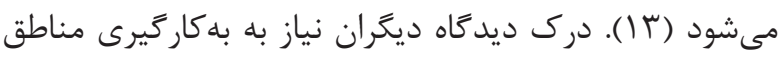

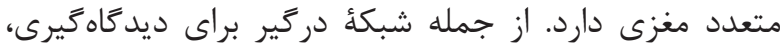

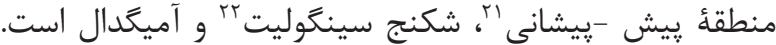

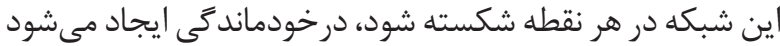

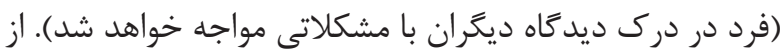

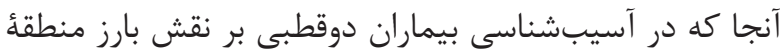

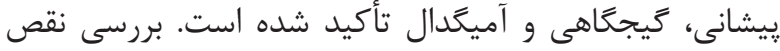

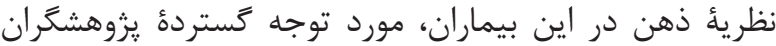

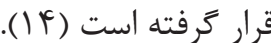
سنجش توانايى نظريئ ذهن در بيماران دوقطبى از نظر بالينى و

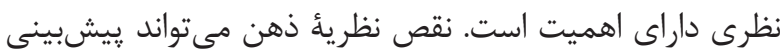

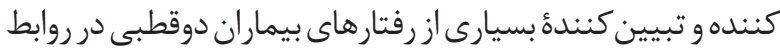
اجتماعى باشد و حتى بسيارى از علايم آنان را توجيه كند (ه (1).

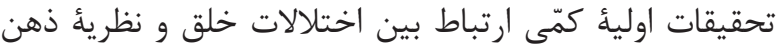

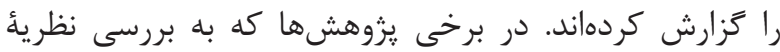

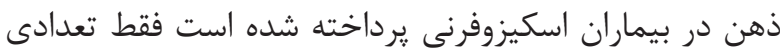

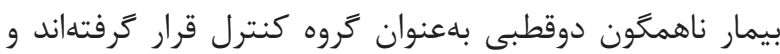

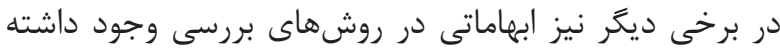

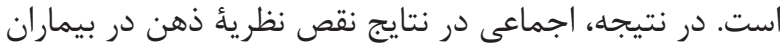

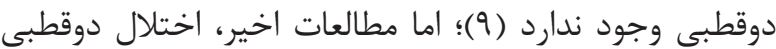

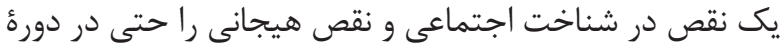

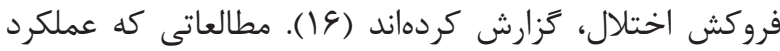

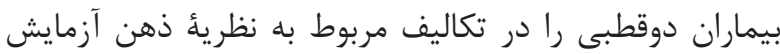

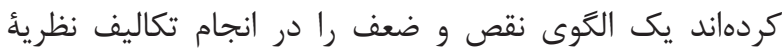

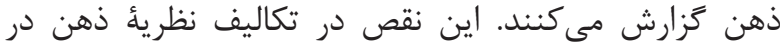

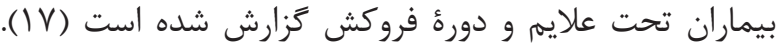

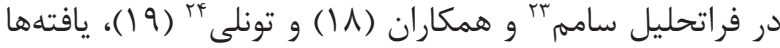

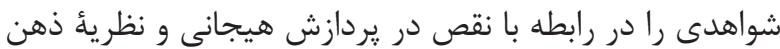

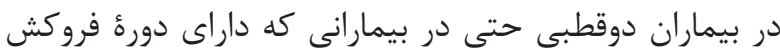

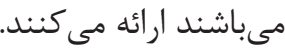

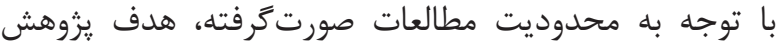

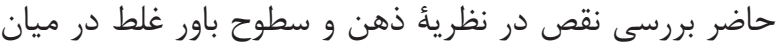

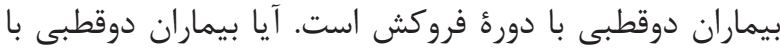

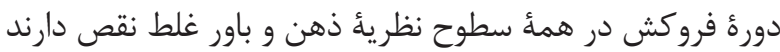
يا در تعدادى از سطوح نقص ديده نوح نظرية ذهن بـون

\footnotetext{
${ }^{25}$ Corcoran

${ }^{26}$ Theory of mind picture stories task

${ }^{27}$ Animation
} 


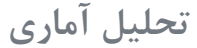

نتايج اين يزوهش در غالب يافتههاى توصيفى و استنباطى ارائه

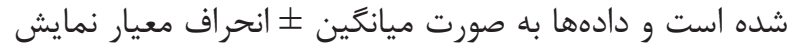

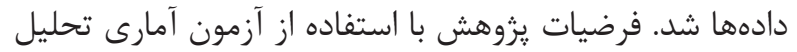

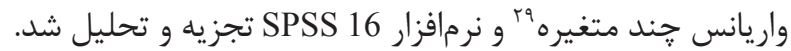

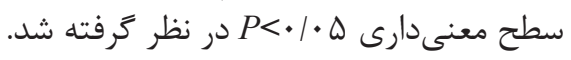

يافتهها

در اين بخش ابتدا اطلاعات جمعيتشناختى و يافتههاى

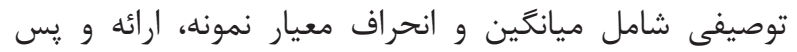

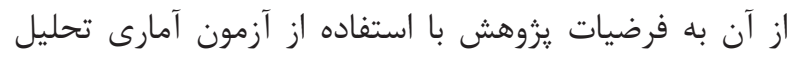

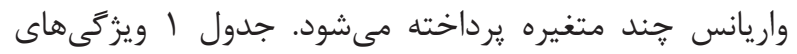

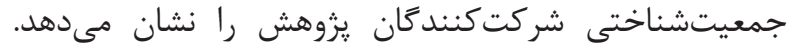

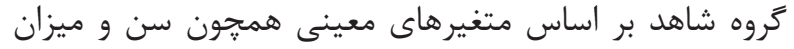

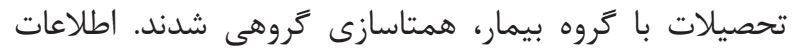

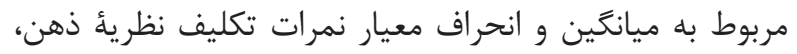

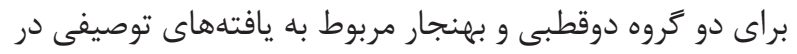
جدول ب آمده است.
در صورت موفقيت آزمودنى در قراردهى كلئ كارتها و وِاسخ

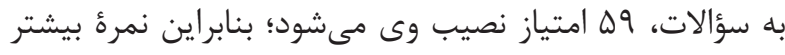

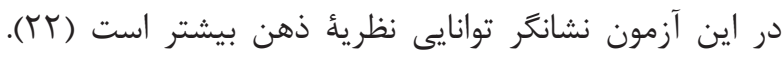

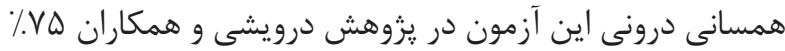

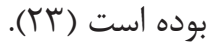

$$
\text { مقياس :ريشانى روانشناختى كسلر }
$$

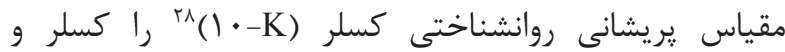

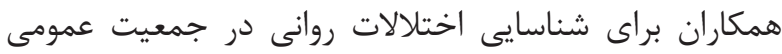

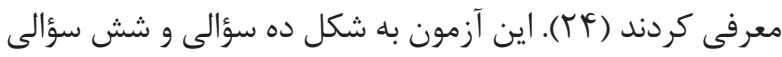

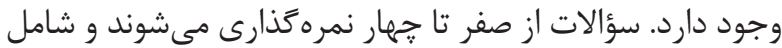

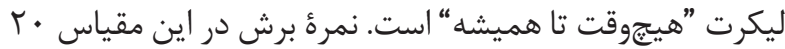

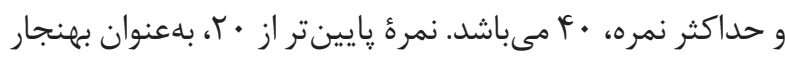

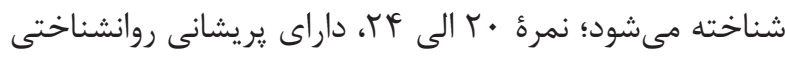

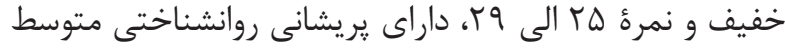

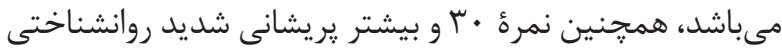

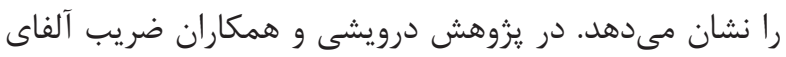

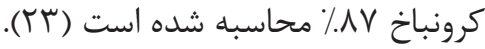

جدول ا- ويزگگىهاى جمعيتشناختى آزمودنىهاى يروهش برحسب جنسيت، تحصيلات و سن.

\begin{tabular}{|c|c|c|c|}
\hline (n-r) & دوقطبى نوع يك با دورة فروكش & & \\
\hline r. & $r$. & مرد & جنسيت \\
\hline ir & IV & كارشناسى & \multirow{3}{*}{ تحصيلات } \\
\hline ir & 1. & ديبيلهم & \\
\hline$\Delta$ & $r$ & زير دييلهم & \\
\hline 10 & 18 & IA-r. & \multirow{3}{*}{ سن } \\
\hline$\Delta$ & 1. & ri-f. & \\
\hline 10 & 8 & f) $-\Delta$. & \\
\hline
\end{tabular}

جدول r- ميانكين و انحراف معيار نمرات تكليف نظريئ ذهن دو كروه شركت كننده در يروهش.

\begin{tabular}{|c|c|c|c|c|}
\hline \multicolumn{2}{|c|}{ تروه هنجار } & \multicolumn{3}{|c|}{ تروه بيمار } \\
\hline انحراف معيار & مياثكين & انحراف معيار & ميائَين & متغيرها \\
\hline$\cdot \pi \cdot \Delta$ & $r / 9$ & $\cdot \pi \cdot \Delta$ & $Y / \Lambda$ & باور غلط سطح اول \\
\hline$\cdot|A|$ & $r / 4$ & $1 / 11$ & $1 / 1$ & باور غلط سطح دوم \\
\hline •/99 & $r / T$ & $1 / \cdot r$ & $1 / N$ & باور غلط سطح سوم \\
\hline$\cdot \pi \cdot \Delta$ & $r / 9$ & $\cdot / \Delta \cdot \Lambda$ & $r / \Delta$ & تعامل و همكارى \\
\hline$\cdot \pi \cdot \Delta$ & $r / 9$ & - /Y१F & $r / T$ & فريب \\
\hline $1 \neq 1$ & $r / 9$ & $\cdot|90|$ & $1 / V$ & كشف فريب \\
\hline
\end{tabular}

${ }^{28}$ Kessler psychological distress scale-10

${ }^{29}$ Multivariable analyze of variance 


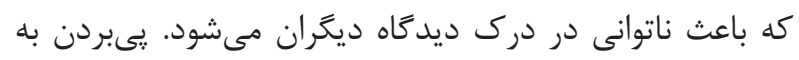

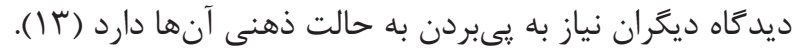

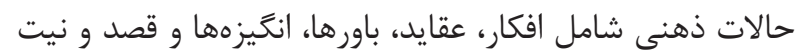

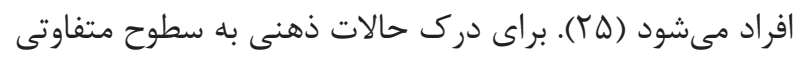

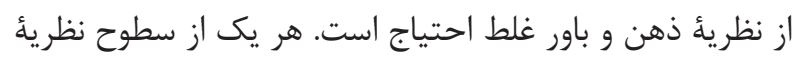

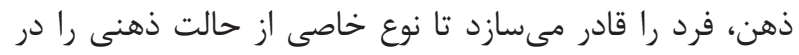

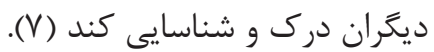

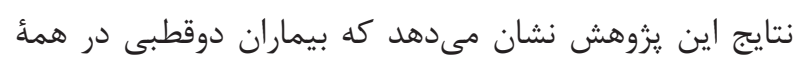

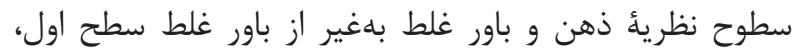

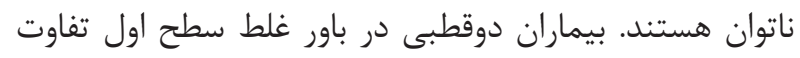

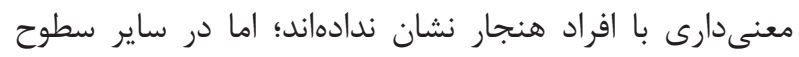

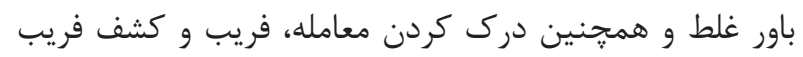

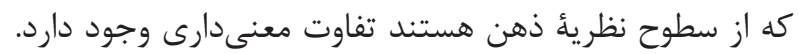

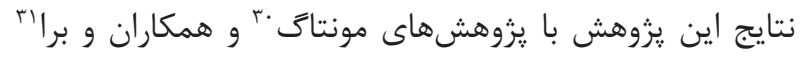

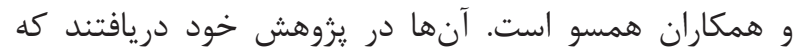

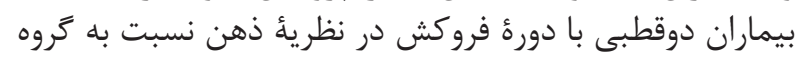

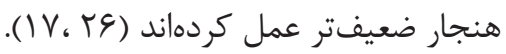

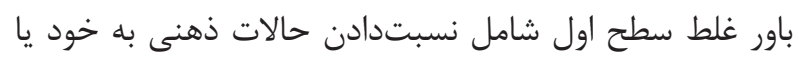

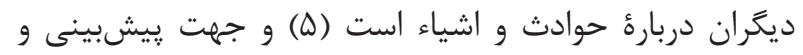

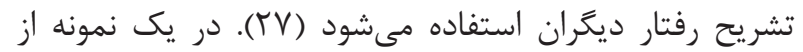

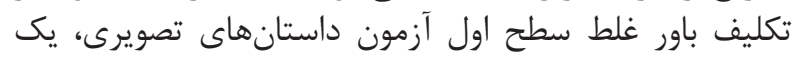

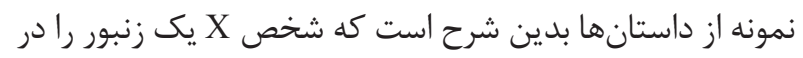

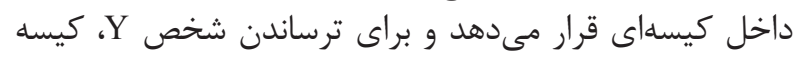

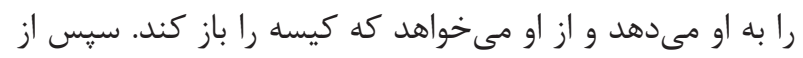

براى بررسى فرضيؤ بروهش مبنى بر نقص نظرئ ذهن نهن در بيماران

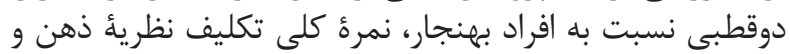

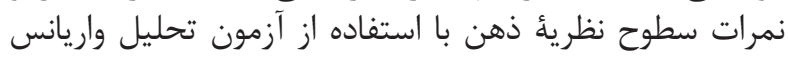

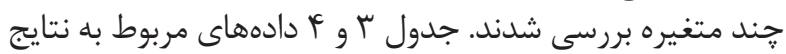

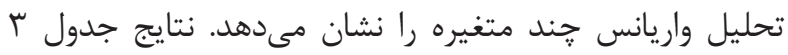

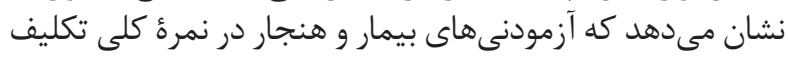

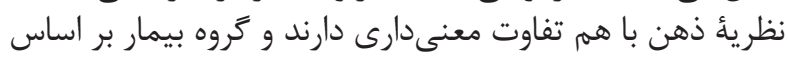

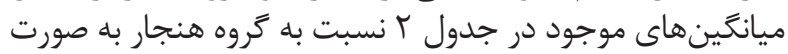

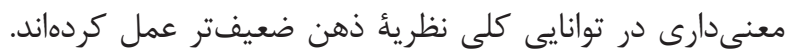

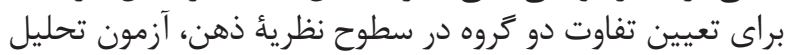

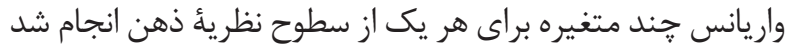

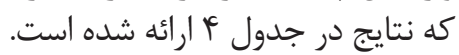

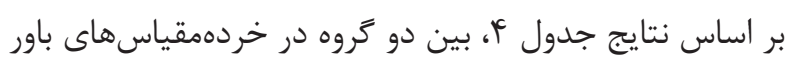

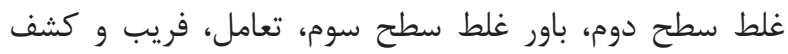

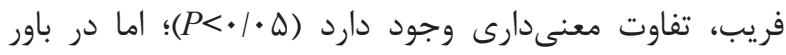

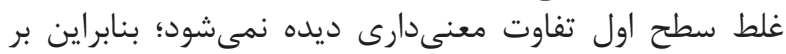

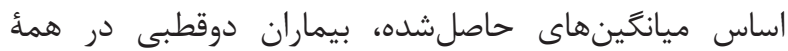

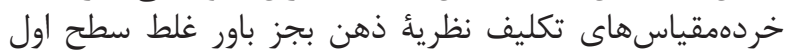

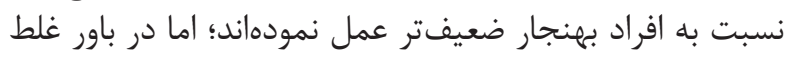

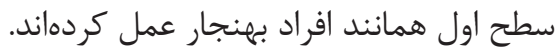

\section{بحث و نتيجه كيرى}

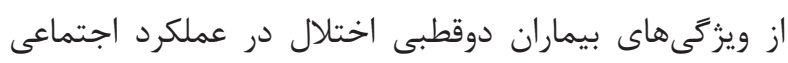

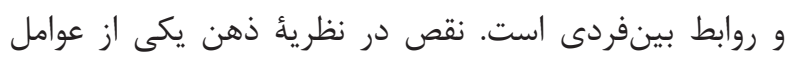

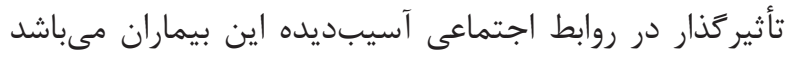

جدول بـ- نتايج آزمون تحليل واريانس حند متغيره اثر بِيلايى نمرة كلى تكليف نظريةٔ ذهن بين دو كروه بيمار و هنجار.

\begin{tabular}{|c|c|c|c|c|c|c|c|}
\hline مجذور اتا & سطح معنى دارى & درجةُ آزادى خطا & درجة آزادى فرضيه & $\mathrm{F}$ & 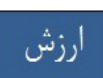 & شاخص & 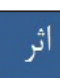 \\
\hline.$/ \pi \Delta \Delta$ &.$|\cdot|$ & - & $4 /$. & $\Delta / \Delta T Y$ & $\cdot / r \wedge \Delta$ & اثر پِيلايى & كروه \\
\hline
\end{tabular}

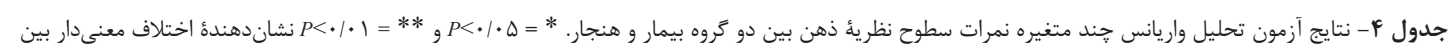

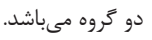

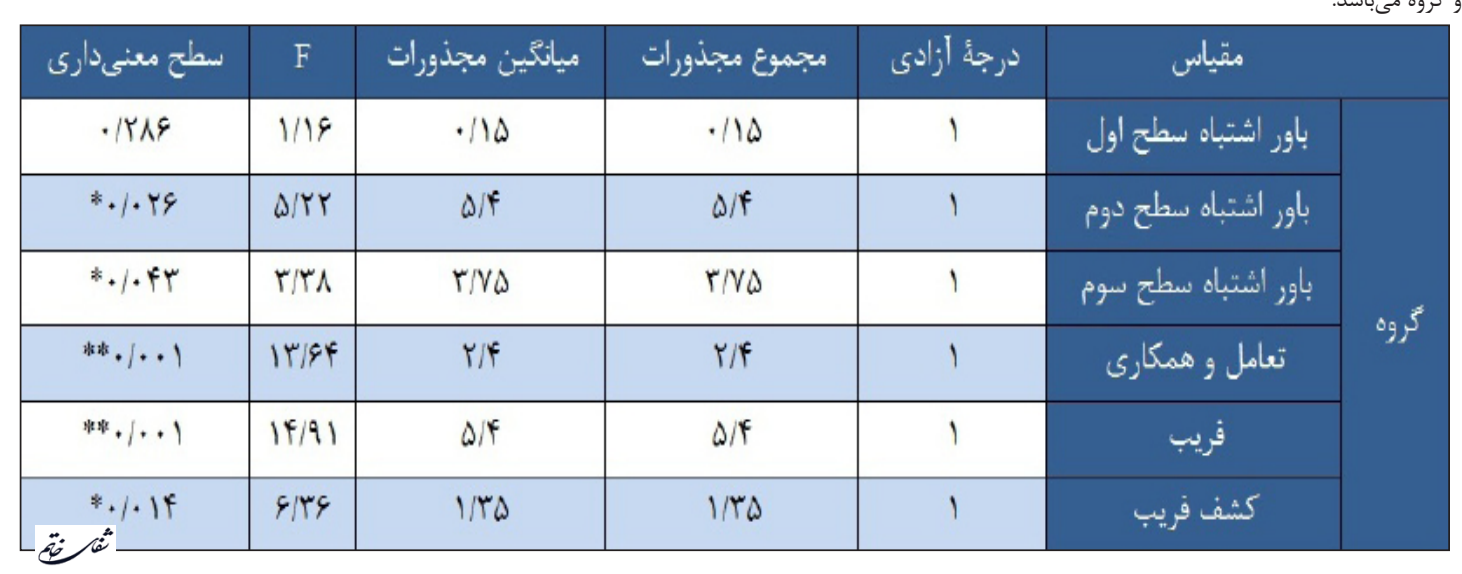


ديد كاه ديكران با استفاده از نظريةٌ ذهن دارد بيماران دوقطبى در

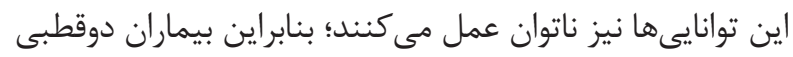

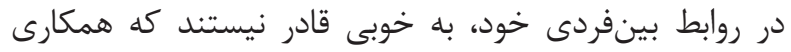

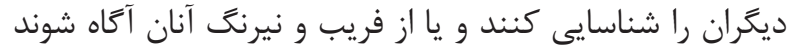

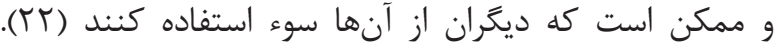

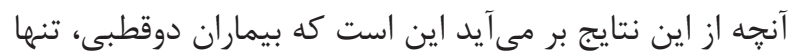

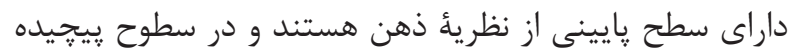

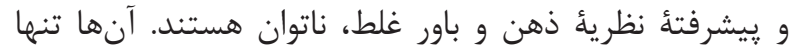

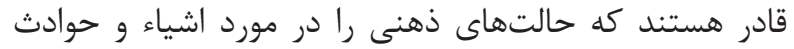

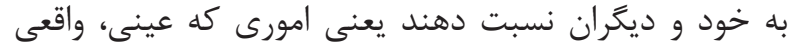

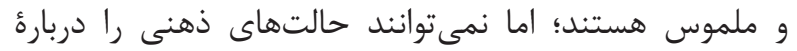

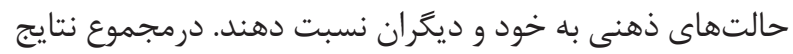

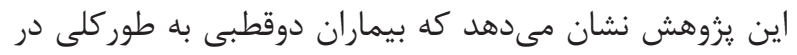

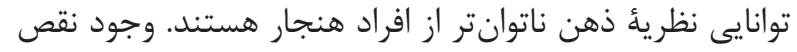

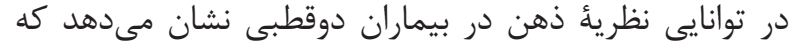

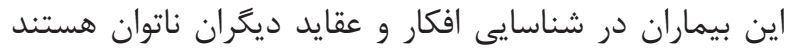

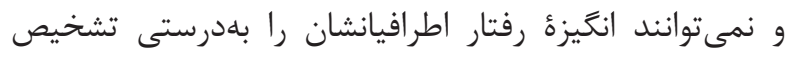

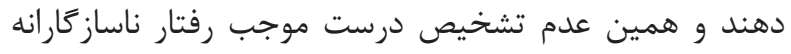

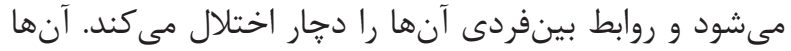

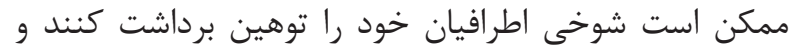

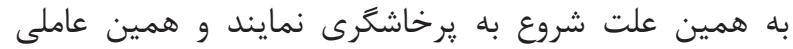

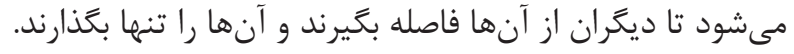

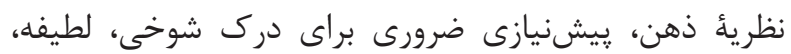

دستانداختن، دروغ و فريب است (• (ץ).

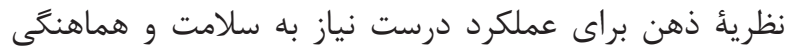

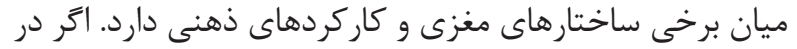

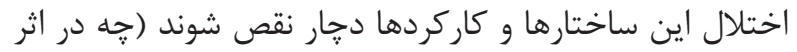

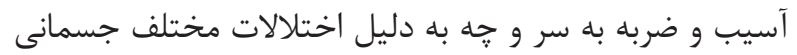

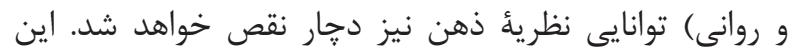

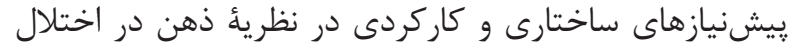

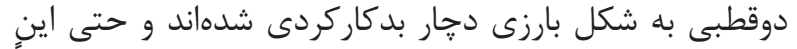

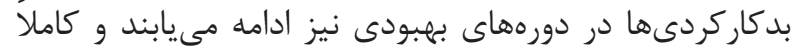

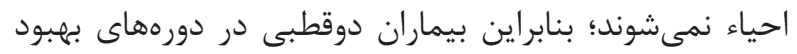
خود در نظرئ ذهن دجار مشكل هستند (r) (I). مطالعات عصبشناختى نيز يافتههاى اين يروهش رائن را تأييد

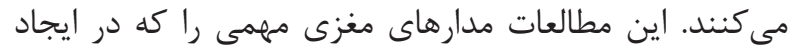

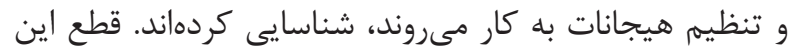

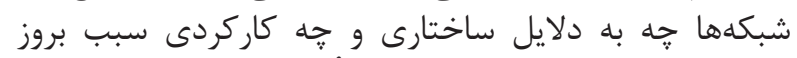

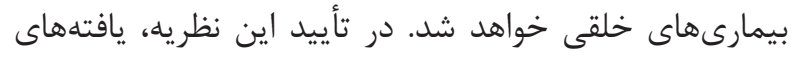

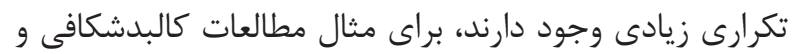

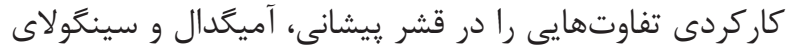

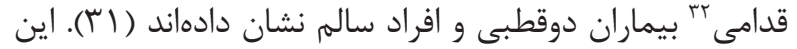

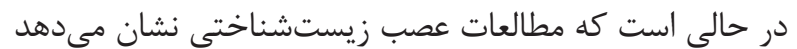

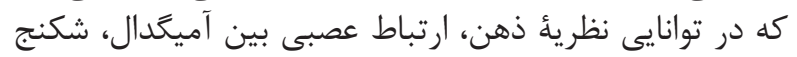

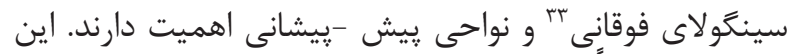

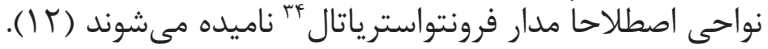

${ }^{32}$ Anterior cingulate

${ }^{33}$ Dorsal cingulate gyrus
آزمودنى يرسيده مىشود كه شخص Y فكر مى كند كه در داخل

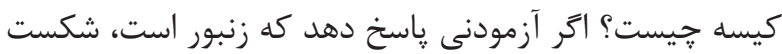

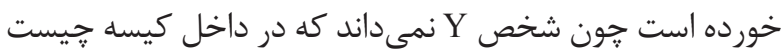

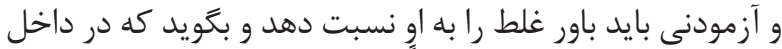

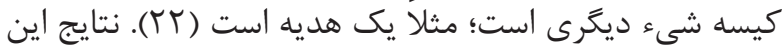

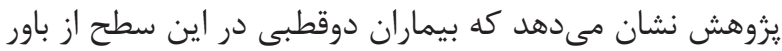

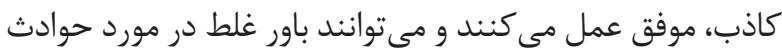

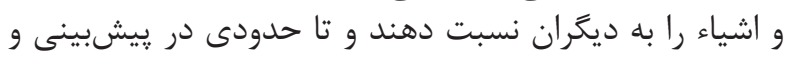
تشريح رفتار ديكران درست عمل كنند. نـان

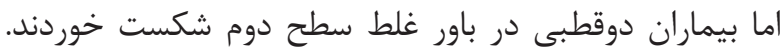

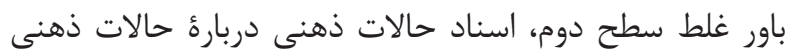

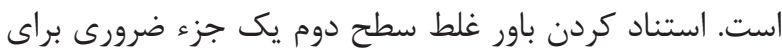

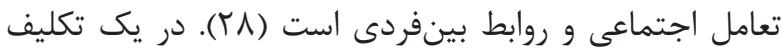

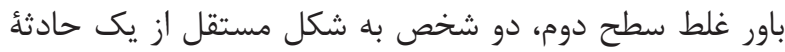

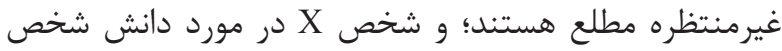

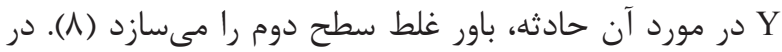

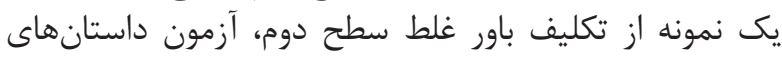

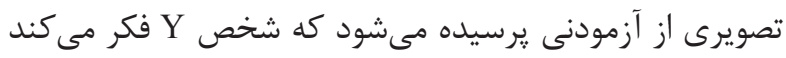

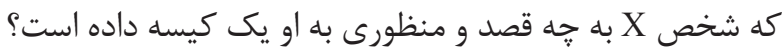

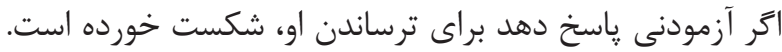

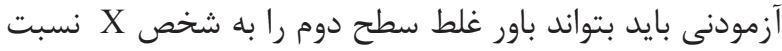

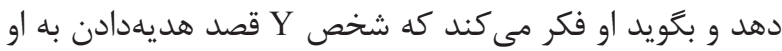

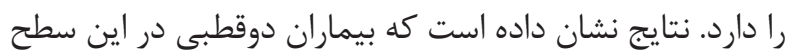

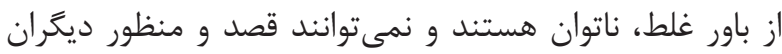

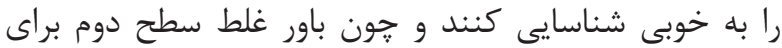

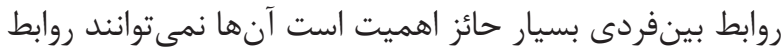

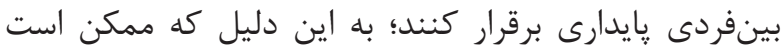

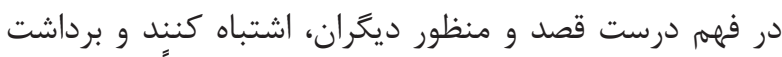

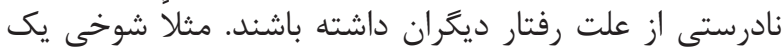

فرد را به اشتباه تمسخر يا توهين برداشت كنند (YT)

همجنين بيماران دوقطبى در باور غلط سطح سوم نيز شكست

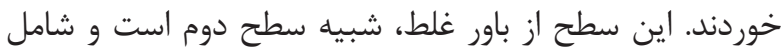

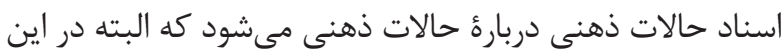

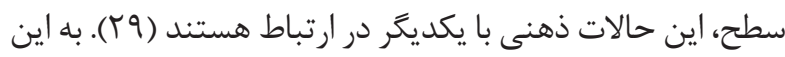

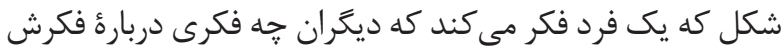

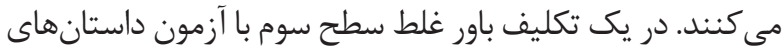

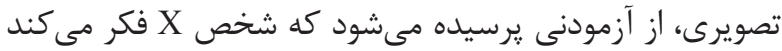

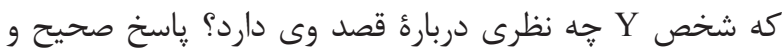

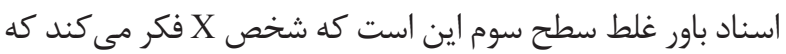

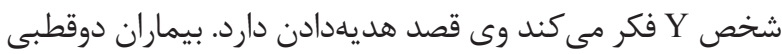

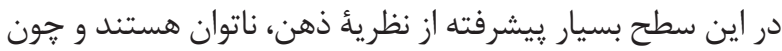

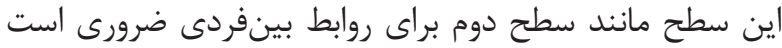

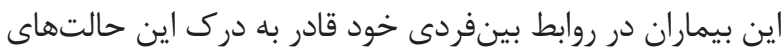

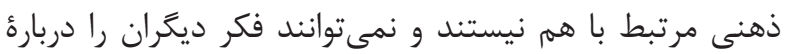

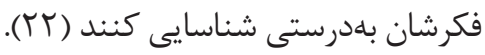
از آنجا كه درك فريب، معامله و كشف فريب نيز نياز به توانايى درى سكى

\footnotetext{
${ }^{34}$ Frontostriatal circuit
} 
مدل كالبدشناسى عصبى تنظيهم خلق، هستند (سب). قشر ييش

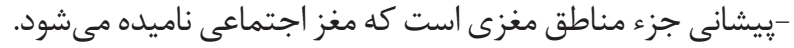

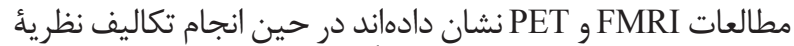

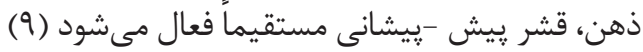
اين يزوهش، همانند ديخر يزوهش هاى حوزهُ روانشناسى و علوم

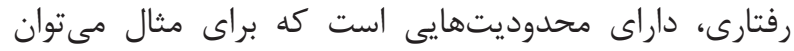

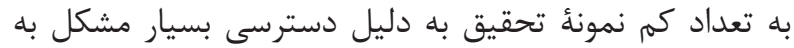

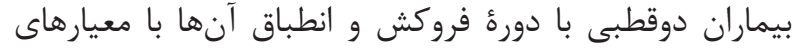

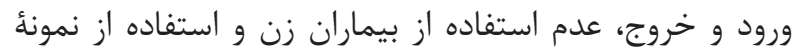

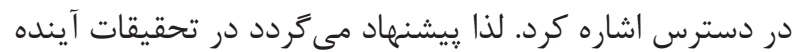

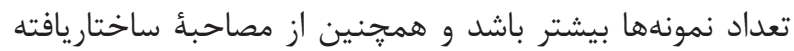

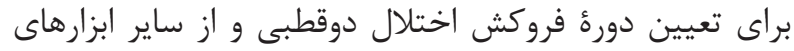

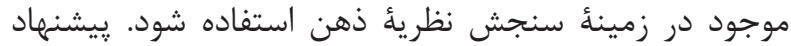

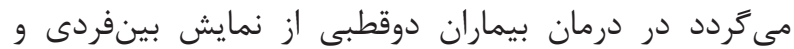

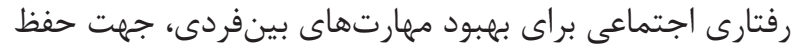

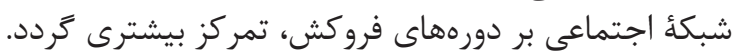

1. Amin Iazdi A. Social cognition and language. Journal of Literature and Humanities Mashhad. 2007; 40(156): 115-34.

2. Premack D, Woodruff G. Does the chimpanzee have a theory of mind? J Behav Brain Sci. 1978; 1(04): 515-26.

3. Baron-Cohen S, O'Riordan M, Stone V, Jones R, Plaisted K. Recognition of faux pas by normally developing children and children with Asperger syndrome or high-functioning autism. J Autism Dev Disord. 1999; 29(5): 407-18.

4. Clements WA, Perner J. Implicit understanding of belief. Cogn Dev. 1994; 9(4): 377-95.

5. Perner J, Wimmer H. "John thinks that mary thinks that..." attribution of second-order beliefs by 5-to 10-year-old children. J Exp Child Psychol. 1985; 39(3): 437-71.

6. Baron-Cohen S, Leslie AM, Frith U. Does the autistic child have a "theory of mind"? Cognition. 1985; 21(1): 37-46.

7. Miller SA. Children's understanding of second-order mental states. Psychol Bull. 2009; 135(5): 749-73.

8. Coonrod EE. Theory of mind, executive function, and social skills in high-functioning children with autism spectrum disorders. PhD Thesis. New York: Vanderbilt University. 2005.

9. Inoue Y, Tonooka Y, Yamada K, Kanba S. Deficiency
شباهت ميان نواحى درگير در نظرئُ ذهن با نواحى درگير در

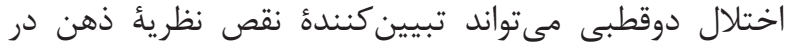

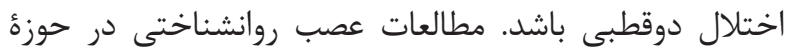

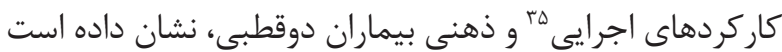

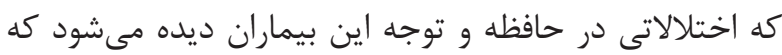

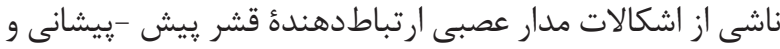

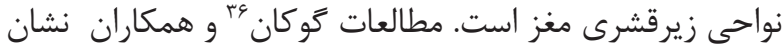

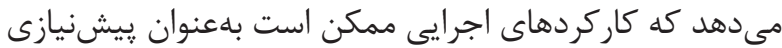

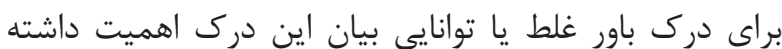

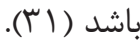

مطالعات اخير نشان مىدهد توانايىهاى شناختى، در تكاليف

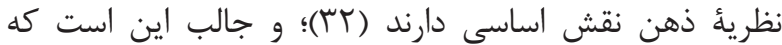

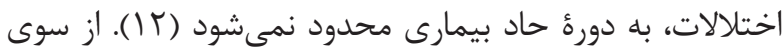

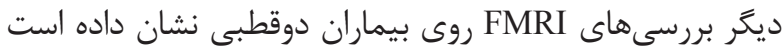

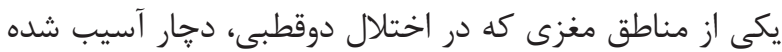

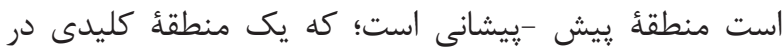

منابع of theory of mind in patients with remitted mood disorder. J Affect Disord. 2004; 82(3): 403-9.

10. Abu-Akel A. The neurochemical hypothesis of 'theory of mind'. Med Hypotheses. 2003; 60(3): 382-6.

11. Purcell AL, Phillips M, Gruber J. In your eyes: Does theory of mind predict impaired life functioning in bipolar disorder? J Affect Disord. 2013; 151(3): 1113-9.

12. Yatham LN, Malhi GS. Bipolar disorder. New York: Oxford University Press. 2010.

13. Fakhari A, Minashiri A, Khazaee S, Movahedi Y, Fallahi A, Taher Panah M. Study of deficiency in theory of mind and emotion regulation in bipolar disorder and depressed patients compared with normal subjects. Urmia Medical Journal. 2014; 25(6): 511-20.

14. Joghataei MMG, Movallali G, Mashhadi A. How our minds can read the minds of others. News Journal of Neuroscience. 2005; 11(3): 677-88.

15. Kerr N, Dunbar RI, Bentall RP. Theory of mind deficits in bipolar affective disorder. J Affect Disord. 2003; 73(3): 253-9.

16. Ibanez A, Urquina H, Petroni A, Baez S, Lopez V, do Nascimento M, et al. Neural processing of emotional facial and semantic expressions in euthymic bipolar (ToM). PLoS One. 2012; 7(10): e46877. doi: 10.1371/ journal.pone.0046877.

17. Montag C, Ehrlich A, Neuhaus K, Dziobek

\footnotetext{
${ }^{35}$ Executive function
}

${ }^{36}$ Goukon 
I, Heekeren HR, Heinz A, et al. Theory of mind impairments in euthymic bipolar patients. J Affect Disord. 2010; 123(1-3): 264-9.

18. Samamé C, Martino D, Strejilevich S. Social cognition in euthymic bipolar disorder: systematic review and meta-analytic approach. Acta Psychiatr Scand. 2012; 125(4): 266-80.

19. Tonelli HA. Cognitive" theory of mind" processing in bipolar disorder. Rev Bras Psiquiatr. 2009; 31(4): 369-74.

20. Delaware A. ENTRY principles and practical research in the humanities and social sciences. Tehran. Publisher Growth. 2013.

21. Corcoran R, Cahill C, Frith CD. The appreciation of visual jokes in people with schizophrenia: a study of 'Mentalizing' ability. Schizophr Res. 1997; 24(3): 319-27.

22. Brüne M. Social cognition and behaviour in schizophrenia. The Social Brain: Evolution and Pathology. 2003: 277-313.

23. Darvishi N, Mahmoud Alilou M, Bakhshipour A, Farnam A, Bahramkhani M. Theory of mind in obsessive-compulsive disorder patients versus normal individuals. Advances in Cognitive Science. 2013; 15(1): 77-89.

24. Kessler RC, Barker PR, Colpe LJ, Epstein JF, Gfroerer JC, Hiripi E, et al. Screening for serious mental illness in the general population. Arch Gen Psychiatry. 2003; 60(2): 184-9.
25. Ansari Najad SM, Adib GN. The effectiveness of teaching the theory of mind on improving students' theory of mind educable mentally retarded. Journal of Clinical Psychology and Counseling. 2011; 2(1): 35-45.

26. Bora E, Vahip S, Gonul A, Akdeniz F, Alkan M, Ogut M, et al. Evidence for theory of mind deficits in euthymic patients with bipolar disorder. Acta Psychiatr Scand. 2005; 112(2): 110-6.

27. Frith U, Happé F. Theory of mind and selfconsciousness: what is it like to be autistic? Mind and Language. 1999; 14(1): 82-9.

28. Tager-Flusberg H, Sullivan K. A second look at second-order belief attribution in autism. J Autism Dev Disord. 1994; 24(5): 577-86.

29. Happé FGE. Understanding minds and metaphors: Insights from the study of figurative language in autism. Metaphor and Symbolic Activity. 1995; 10(4): 275-95.

30. Yaghob Nejad S, Modir Khazani SM. Social cognition in children with down syndrome. Special Education. 2013; 7(120): 37-49.

31. Goukon A, Kikuchi T, Noguchi K, Ohuchi T, Hirano M, Hosokawa T. Developmental order driving the relationship between executive functions and theory of mind: a case study. Psychol Rep. 2006; 98(3): 662-70.

32. Bull R, Phillips LH, Conway CA. The role of control functions in mentalizing: dual-task studies of theory of mind and executive function. Cognition. 2008; 107(2): 663-72.

33. Taylor EH. Atlas of bipolar disorders (encyclopedia of visual medicine series) $1^{\text {st }}$ ed. CRC Press. 2006. 